\title{
Health numeracy: Perspectives about using numbers in health management from African American patients receiving dialysis
}

\author{
Julie A. WRIGHT NUNES,' Chandra Y. OSBORN, ${ }^{2,3}$ T. Alp IKIZLER, ${ }^{4,5}$ \\ Kerri L. CAVANAUGH ${ }^{4,5}$ \\ 'Department of Internal Medicine, Division of Nephrology, University of Michigan Health System, Ann \\ Arbor, Michigan; Departments of ${ }^{2}$ Medicine, Division of General Internal Medicine \& Public Health, \\ ${ }^{3}$ Biomedical Informatics, ${ }^{4}$ Medicine, Division of Nephrology, ${ }^{5}$ Vanderbilt Center for Kidney Disease, \\ Vanderbilt University Medical Center, Nashville, Tennessee, USA
}

\begin{abstract}
Health numeracy is linked to important clinical outcomes. Kidney disease management relies heavily on patient numeracy skills across the continuum of kidney disease care. Little data are available eliciting stakeholder perspectives from patients receiving dialysis about the construct of health numeracy. Using focus groups, we asked patients receiving hemodialysis open-ended questions to identify facilitators and barriers to their understanding, interpretation, and application of numeric information in kidney care. Transcripts were analyzed using content analysis. Twelve patients participated with a mean (standard deviation) age of 56 (12) years. All were African American, $50 \%$ were women, and $83 \%$ had an annual income $<\$ 20,000 /$ year. Although patients felt numbers were critical to every aspect in life, they noted several barriers to understanding, interpreting and applying quantitative information specifically to manage their health. Low patient self-efficacy related to health numeracy and limited patient-provider communication about quantitatively based feedback, were emphasized as key barriers. Through focus groups of key patient stakeholders we identified important modifiable barriers to effective kidney care. Additional research is needed to develop tools that support numeracy-sensitive education and communication interventions in dialysis.
\end{abstract}

Key words: Health numeracy, quantitative health literacy, dialysis, kidney disease, disparities

\section{INTRODUCTION}

Health literacy is a term used to describe "the degree to which individuals have the capacity to obtain, process,

Correspondence to: J. A. Wright Nunes, MD, MPH, Department of Internal Medicine, Division of Nephrology, University of Michigan Health System, Simpson Memorial Building, Room 311, 102 Observatory, Ann Arbor, MI 48109, USA. E-mail: juwright@med.umich.edu and understand basic health information"1 and has been associated with clinical outcomes across health conditions. ${ }^{2}$ When measured in patients, health literacy has often been assessed as print or prose literacy. The impact of low quantitative literacy (i.e., numeracy) on clinical outcomes is largely unexplored. A revision to the definition of health literacy has been proposed as an operational framework for the construct of health numeracy: "the degree to which individuals have the capacity to access, process, interpret, communicate, and act on numerical, 
quantitative, graphical, biostatistical, and probabilistic health information needed to make effective health decisions." Recent literature suggests health numeracy is equally important to health literacy. Limited numeracy is associated with patient difficulty understanding health risks and treatment options, and lower adherence to selfmanagement of health conditions. ${ }^{3}$

Limited health numeracy may play a role in suboptimal health outcomes in patients with kidney disease. ${ }^{4}$ Kidney disease is a sentinel example of a chronic disease that relies heavily on patient numeracy skills for effective management across the continuum of its severity. Largely asymptomatic until advanced, it is often only through laboratory testing with numeric-based results (e.g., estimates of Glomerular filtration rate or creatinine clearance) that kidney disease is identified. Moreover, monitoring for complications and therapy efficacy depend on the evaluation and interpretation of quantitative laboratory and clinical results. If a patient's ability to interpret numeric information is limited, he/she may find it difficult to engage in conversations and decision making with providers about "their numbers." This difficulty is likely exacerbated as kidney failure ensues, with even more demands placed on patients once they are receiving dialysis. Little is known from the patient's perspective about the use of clinical numeric information in dialysis-related kidney care. This information is critical to augment kidney disease educational programs aimed to optimize management and adherence in this high-risk patient population.

We performed a qualitative study to gain insight from patients about the potential role of health numeracy in the delivery of health information. Using patient-centric focus groups we asked patients receiving hemodialysis how they process, understand, and utilize numeric information dayto-day in their health management. We also asked them about their perceived self-efficacy using numbers, and about barriers and facilitators to applying numeric information to manage kidney health. We hypothesized that patients would find kidney-related numerical information as a significant barrier to the transmission of important educational components and clinical recommendations of care.

\section{METHODS}

We conducted focus groups with patients receiving chronic hemodialysis. Each focus group was designed to include three to five patients and used convenience sampling to recruit from two outpatient hemodialysis centers. From May to July 2008, patients were recruited from dialysis facilities consisting of a population that is $51 \%$ female and 69\% African American, with a mean (standard deviation [SD] ) age of 54 (16) years. Study inclusion criteria were: ability to speak English, age 18 years or older, and ability to provide written informed consent.

Focus group sessions were held in conference rooms within dialysis centers. No medical personnel were allowed into these rooms in an effort to facilitate open and honest communication. Participants completed a demographic survey and participated in moderated discussion about numeracy and its applications (for the focus group questions, see Appendix S1) Sessions lasted 60-90 minutes and were digitally audio-recorded and transcribed verbatim. The number of focus group sessions corresponded to the amount needed to reach thematic saturation, which is important in qualitative research about a new topic or phenomenon. ${ }^{5}$

Transcripts were entered into QSR NVivo version 8.0 (QSR International Pty., Ltd. Doncaster Victoria, Australia), a software program for storage, coding, and analyzing qualitative data. Transcripts were reviewed using the method of analytic induction and comparative analysis methods. ${ }^{6}$ Analytic induction involves scanning qualitative data for themes or categories, developing a working scheme after examination of initial cases, and then modifying the scheme for subsequent analyses. ${ }^{7}$ The first author (JWN) reviewed transcripts of interviews line by line to determine emerging themes, and develop an initial coding scheme by which transcripts were coded preliminarily. Then two authors (JWN, KC) met and reviewed the preliminary coding of each transcript and resolved disagreements by consensus.

The final coding scheme and transcript codes were then entered into NVivo version 8 with a quantitative assessment of numbers of comments in content areas performed, along with a descriptive analysis. Previous research suggests the frequency of participants' comments correlates with measures of importance, and this was used to prioritize themes. ${ }^{8,9}$ The Institutional Review Board at the Vanderbilt University Medical Center approved all study procedures prior to participant enrollment and participants completed informed consent with knowledge that anything shared in the focus group sessions would not be shared with clinical personnel/physicians at their dialysis center.

\section{RESULTS}

Thematic saturation was reached after three focus group sessions, involving a total of 12 participants (Table 1). The mean (SD) age was 56 (12) years, 50\% were female, and all were African American. All participants attended high 
Table 1 Participant characteristics

\begin{tabular}{ll}
\hline Characteristic $(\mathrm{N}=12)$ & $\begin{array}{c}\text { Mean }(\mathrm{SD}) \\
\text { or } \mathrm{n}(\%)\end{array}$ \\
\hline Age (years) & $56(12)$ \\
Female & $6(50 \%)$ \\
African American & $12(100 \%)$ \\
Income $<20,000$ per year & $10(83 \%)$ \\
Formal education/highest number of years & \\
$\quad$ completed* & \\
10 & $1(8 \%)$ \\
11 & $3(25 \%)$ \\
12 & $4(33 \%)$ \\
14 & $1(8 \%)$ \\
15 & $2(17 \%)$ \\
16 & $1(8 \%)$ \\
Married & $4(33 \%)$ \\
Employed outside the home & $1(8 \%)$ \\
Years on dialysis & $9(9)$ \\
Self-rated health fair or poor (selections were: & $8(66 \%)$ \\
$\quad$ poor, fair, good, very good, or excellent) & \\
\hline
\end{tabular}

* Patients were asked to report the number of years they attended formal education, through year 12 considered high school graduate. $\mathrm{SD}$, standard deviation.

school, with the majority graduating and some obtaining more formal education after high school. However, most were unemployed, and $83 \%$ reported household annual incomes of $<\$ 20,000$. Participants had been receiving dialysis treatments for a mean (SD) of 9 (9) years. Sixty-six percent of patients rated their health status as less than "good."

Interviews revealed 356 total statements with three major themes: (1) acquisition and mastery-how patients learned to use quantitative health information; (2) application-how patients use numbers in health care; and (3) attitude and self-efficacy, which describes the importance patients placed on numerical information and their own confidence using numbers in health care (Figure 1). Discussion centered on kidney care. However, additional comments revealed how participants use numbers outside of kidney care and about resources patients use to interpret and apply numbers to their care.

Participants discussed facilitators and barriers within each theme. Facilitators included tools and resources patients felt helped them apply numbers in care. Barriers largely related to perceived system (e.g., limited educational support) and personnel (e.g., limited patientprovider communication) issues. Representative comments are summarized in Table 2, along with the number of total statements related to each theme (i.e., frequency). Quotations shown later are presented using age, sex, and dialysis vintage as references.

\section{Acquisition and mastery of numbers}

Acquisition and mastery of numbers encompassed 116 patient statements. Here participants discussed facilitators of and barriers to learning how to interpret and apply numbers within their medical care. They also described their current fund of knowledge related to numbers, often listing their experiences using numbers outside of medical care as the foundation for their numeracy skills. They expressed difficulty using these same skills in the context of medical care. Patients discussed novel pathways leading to numerical understanding, for example, through recreational activities. A significant number of statements from one participant described the complex application and interpretation of numbers acquired through calculating odds in "counting."

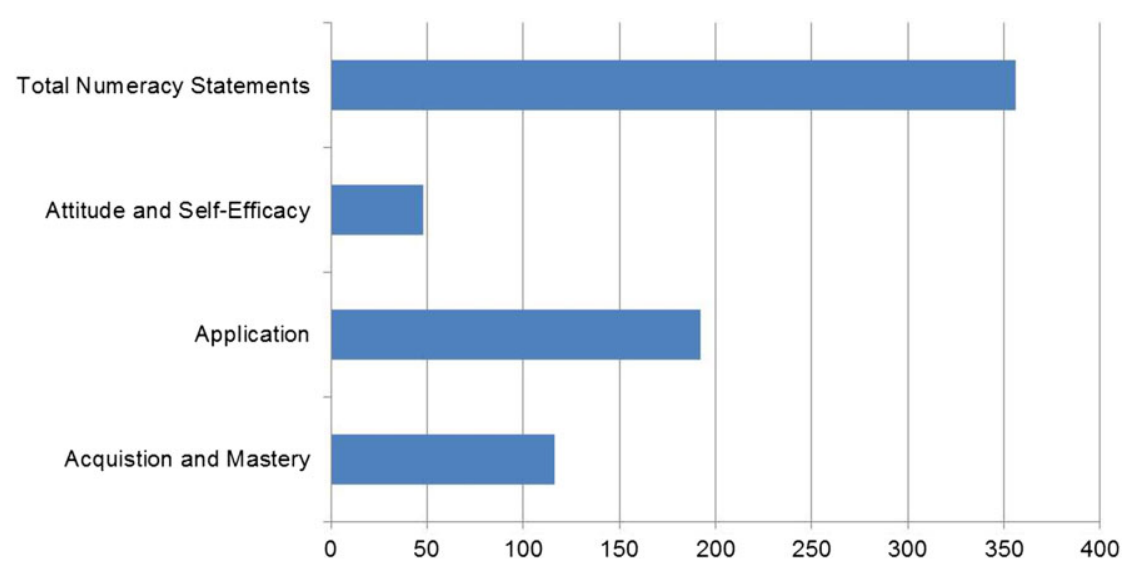

Figure 1 Focus groups summary: Frequency of statements for each theme and total. 
Table 2 Focus group themes and representative comments

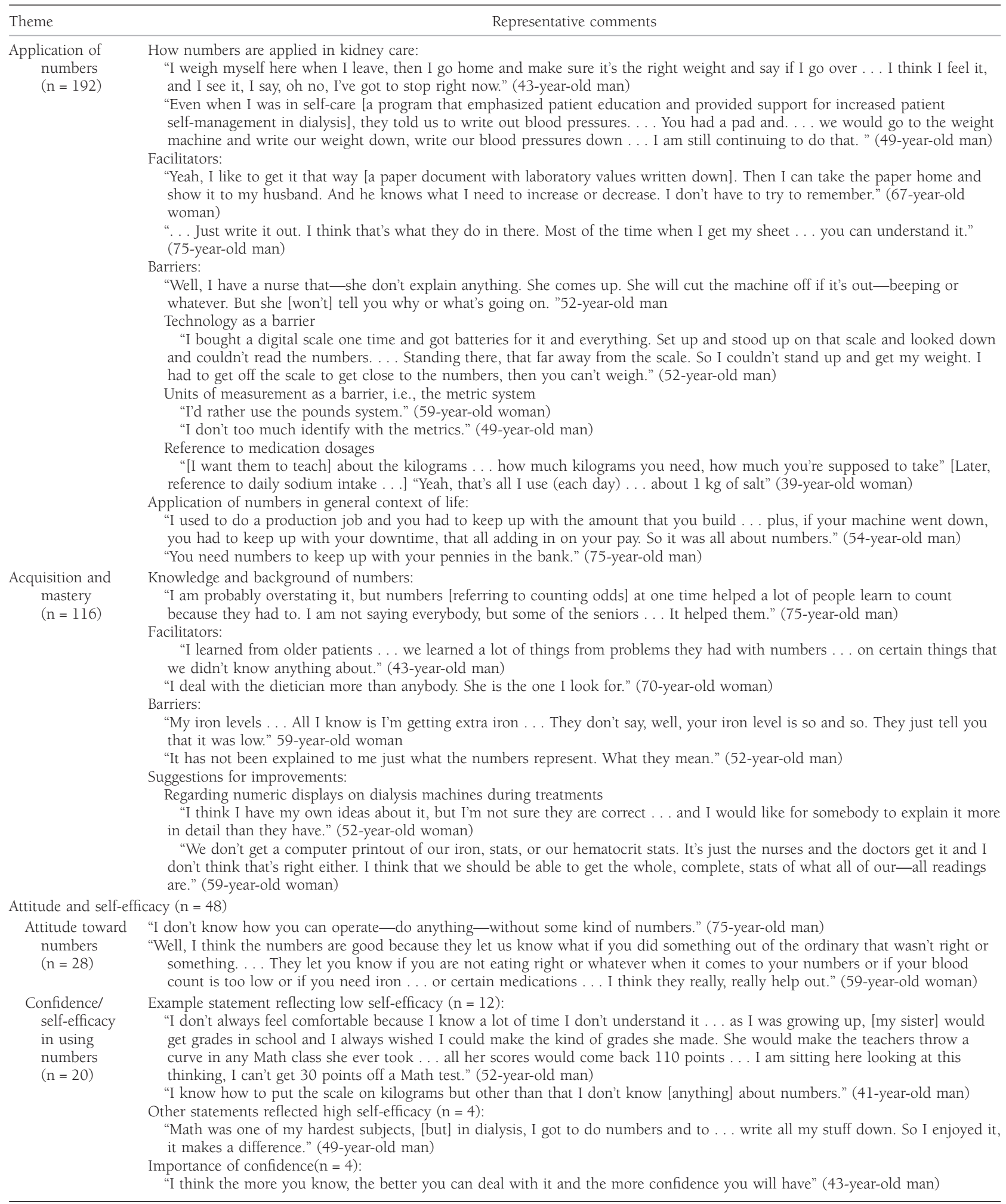

$\mathrm{n}=$ statement frequency 
I am probably overstating it, but numbers [referring to counting odds] at one time helped a lot of people learn to count because they had to. I am not saying everybody, but some of the seniors. . . . It helped them. (75-year-old man, 11 years dialysis)

Perceived facilitators also included people i.e., family members and medical staff. Barriers focused on lack of communication, particularly a need for more assistance with interpreting quantitative feedback from medical staff.

It has not been explained to me just what the numbers represent. What they mean. (52-year-old man, 2 years dialysis)

Participants offered suggestions on how using numbers might be made easier with more one-on-one discussion.

[Regarding numeric displays on dialysis machines during treatments] I think I have my own ideas about it, but I'm not sure they are correct ... and I would like for somebody to explain it more in detail than they have. (52-year-old woman, 3 years dialysis)

\section{Application}

Application of numbers included 192 participant statements and described how patients use numbers within medical care. In dialysis care, patients used health numeracy skills to monitor their weight, check fluid removal during dialysis, and assess their blood pressure. Participants described adjusting their fluid intake and medication regimens based on their interpretation of these values.

I weigh myself here when I leave, then I go home and make sure it's the right weight and say if I go over ... I think I feel it, and I see it, I say, oh no, I've got to stop right now. (43-year-old man, 33 years dialysis)

Even when I was in self-care [a program that emphasized patient education and provided support for self-management], they told us to write out blood pressures .... You had a pad and ... we would go to the weight machine and write our weight down, write our blood pressures down. . . . I am still continuing to do that. (49-year-old man, 10 years dialysis)

Patients perceived facilitators largely as technology and tools (e.g., scales, measuring cups, calculators, pencil and paper). Patients emphasized a need for a plain and uncomplicated presentation of testing results and pre- ferred written materials handed to them. Specifically, when asked about a preference for graphical displays of numbers vs. other methods, participants had a preference for numbers written down or listed on paper rather than graphical displays.

Yeah, I like to get [a paper document with laboratory values written down]. Then I can take the paper home and show it to my husband. And he knows what I need to increase or decrease. I don't have to try to remember. (67-year-old woman, 4 years dialysis)

... Just write it out. I think that's what they do in there. Most of the time when I get my sheet ... you can understand it. (75-year-old man, 11 years dialysis)

Interestingly, only one participant commented that computers and information from the Internet was helpful. This was not supported by other comments in any of the focus groups.

Barriers to applying numbers in kidney care were led by the perception that medical staff did not take time to explain things. During dialysis, there were often alarms indicating a numeric parameter out of "normal range." Patients wanted to understand the meaning of these alarms, and how to interpret them.

Well, I have a nurse that-she don't explain anything. She comes up. She will cut the machine off if it's out-if its beeping or whatever. But she [won't] tell you why or what's going on.

(52-year-old man, 2 years dialysis)

While technology and tools were promoted to patients as potential facilitators to monitor progress, patients voiced concerns that many tools were not helpful when they did not accommodate other physical conditions (e.g., poor vision).

I bought a digital scale one time and got batteries for it and everything. Set up and stood up on that scale and looked down and couldn't read the numbers. ... Standing there, that far away from the scale. So I couldn't stand up and get my weight. I had to get off the scale to get close to the numbers, then you can't weigh. (52-year-old man, 2 years dialysis)

The metric system was also confusing-in particular, related to medication dosing and dietary nutrient restrictions.

I don't . . . identify with the metrics. (49-year-old man, 10 years dialysis) 
[About medication dosages] [I want them to teach] about the kilograms... how much kilograms you need, how much you're supposed to take [Then, a later reference to dietary sodium intake .... Yeah, that's all I use [each day] ... about 1 kilogram of salt. (39-year-old woman, 5 years dialysis)

Although the focus group moderator's questions were clinically oriented, it is important to note that patients often gave examples about using numbers outside of medical care. Patients described using numbers in money management, managerial job duties, and even recreational activities (e.g., sports-interpreting probabilities).

\section{Attitude and self-efficacy}

Patients provided insightful reflection with 48 statements.

Patients felt numbers and their application were of utmost importance in health care and in life. One participant stated a person could not "operate" or "do anything" without the involvement of "some kind of numbers."

They let you know if you are not eating right or whatever when it comes to your numbers or if your blood count is too low or if you need iron ... or certain medications. ... I think they really, really help out. (59-year-old woman, 19 years dialysis)

Lastly, patients described confidence (self-efficacy) using numbers. Efficacy statements were mixed, reflecting low and high self-efficacy. Some statements described the importance of self-efficacy to impact a person's ability to successfully use numbers. Generally, most statements focused on low self-efficacy.

I don't always feel comfortable because I know a lot of time I don't understand it ... as I was growing up, [my sister] would get grades in school and I always wished I could make the kind of grades she made. She would make the teachers throw a curve in any math class she ever took ... all her scores would come back 110 points ... I am sitting here looking at this thinking, I can't get 30 points off a math test. (52-year-old man, 2 years dialysis)

I know how to put the scale on kilograms, but, other than that, I don't know (anything) about numbers. (41-year-old man, 5 years dialysis)

Patients noted their own difficulty in talking about numeracy as a construct of medical care within the focus groups.
Well, I have heard that these talks [focus groups] don't go too good with numbers. ... I heard other people speaking about these meetings and they said ... "I don't know anything about numbers."

(52-year-old man, 2 years dialysis)

\section{DISCUSSION}

To our knowledge, this is one of a few studies, if any, eliciting stakeholder perspectives from patients receiving dialysis about the construct of health numeracy. Our research is especially relevant given the numeracy demands placed on these highly vulnerable patients receiving intensive medical therapy who are often provided with clinical feedback that is largely quantitative. Our focus groups brought to light specific and valuable insights. Understanding numbers, their meaning and interpretation especially in end-stage kidney care was seen as critical and desired by all patients. Yet, fundamental concerns exist about understanding basic quantitative concepts, particularly when applying numbers to individual health-care needs.

Patients described their own as well as provider and system barriers to interpreting and applying numeric information in care management. Provider barriers focused on the lack of time providers spent explaining numeric information and its relevance to patients individually. There was no discussion about targeted education addressing interpretation and understanding. Patients felt that concepts like units of measurement, medication dosages, and laboratory parameters were confusing. Some patients referred to daily nutrient and medication units using kilograms.

Providing targeted education that addresses the needs of each patient may be challenging. Receiving printed materials along with individualized information by providers were perceived as facilitators in our study, but patients did not feel additional graphic displays were helpful. Although "pictographs" have been used to augment health communications for patients about disease risks, successful interpretation of icon types depends on numeracy skills. ${ }^{10}$ A conceptual model has been proposed to help providers overcome challenges that arise when discussing and sharing quantitative health information with patients-including the use of an algorithmic approach that matches patients' numeracy skills with the information they are being given. ${ }^{11}$ In addition, many of the facilitators brought up by patients in our study have also been promoted as techniques that can optimize patient-provider communication about quantitative health information-and include keeping the 
presentation of information simple, formatting messages for clarity, removing nonessential/distracting information, confirming comprehension, and reframing information to patients. ${ }^{11}$

Another key theme that emerged from our study was low patient self-efficacy with interpreting and applying numbers in kidney care. This was in contrast to experiences patients provided outside of care. Interpretive numeracy (understanding strengths and limitations of numbers in relation to disease, treatment efficacy, and outcomes $)^{12}$ is suggested as the highest and most abstract level of quantitative skill. Interestingly, our patients provided examples of using a similar skills set in a variety of activities outside medicine (professional employment, money management, recreation), but expressed difficulty using these same skills in dialysis care. The reason for this is unclear. However recent research suggests health numeracy is not the same as general numeracy; and overall, people perform significantly worse when asked numerically related questions in the context of health domains as compared with either pure math or financial domains. ${ }^{13}$ This may provide some explanation for the difficulty patients noted when using and interpreting numbers in kidney care and even with talking about the construct of numeracy within a medical context.

There are limitations to our study. The sample size was small, of older age, one race, and was predominantly of low socioeconomic status. It is difficult to surmise preferences in all patients when exposure to specific alternatives may be limited within a subset of the entire population. Although our sample size was small, past exploratory research identifies similar numbers as sufficient to identify critical themes, and $92.2 \%$ of codes when compared with larger samples, especially where higher-level concepts are concerned. ${ }^{5}$ The characteristics of our patients did not entirely represent the patient population of the dialysis centers we recruited from, or the dialysis US population as a whole. ${ }^{14}$ Our patients were older and all were African American. There could have been potential for this to limit the emergence of new themes that would have otherwise been brought forth in a more heterogeneous population (e.g., patients of different races or of higher socioeconomic status). However, our study provides a unique opportunity to highlight perspectives about barriers to using complex health information from patients shown to be most vulnerable to kidney disease complications ${ }^{15}$ and mortality, ${ }^{16}$ and additionally, offers important information from a patient population that is not well represented in research. Lastly, qualitative interpretation may be subject to potential influences of prior work of the research team. ${ }^{17}$ Our analysis plan was designed to encourage emergence of new themes, ${ }^{18}$ and the team included members from a range of disciplines (medicine, psychology) including some who had never worked in the area of numeracy or kidney disease.

Still, this research has important implications. Only one patient in our study suggested the use of computers or the Internet to support their health-care health numeracy needs. This was lower than expected based on surveys conducted about Internet use in dialysis patients. ${ }^{19}$ This may reflect a "digital divide" as most of our patients were older and may use technology less than their younger counterparts. ${ }^{20,21}$ Yet, technology and digital media are increasingly championed as important resources for expanding and enriching patient self-management and education. ${ }^{22,23}$ Based on feedback from our focus groups, a critical part of meeting patients' numeracy needs includes appropriately matching communications with an individualized presentation of patient-specific quantitative health information, which may need to include less use of technology rather than more.

Another important implication relates to patient selfefficacy using and applying numbers in kidney care. Increased self-efficacy is associated with patients having more knowledge about their disease ${ }^{24}$ and increased selfmanagement behaviors. ${ }^{25}$ Most comments in our focus groups centered on low self-efficacy. Health coaching may help with this. Health coaching uses positive psychology, motivational interviewing, and goal setting to help patients better cope with managing their illness and engage in care with their providers. ${ }^{26,27}$ Perhaps programs that use health-coaching principles could be applied specifically to increase patient self-efficacy in the health numeracy domain. Although provider time and resources in health care are limited, current changes in CMS reimbursement include education benefits for patients with kidney disease. Interventions that include coaching to increase self-efficacy prior to providing patients with disease-specific education may serve as a powerful adjunct to these educational benefits as well. ${ }^{28}$

Although our study expands upon an area of research in kidney disease where little prior work has been done, admittedly, there is more to do. More information is needed pertaining to specifics about how we can improve health-related communication for our patients. Determining not only what information patients want, but the context in which they want it is critical to augment current communication efforts and eliminate anxiety provoked by numeracy barriers.

In conclusion, through our patient-centric study, we obtained specific and important insights into the facilitators of and barriers to patients' applying numeric 
information in kidney disease care. They identified potential modifiable targets for future interventions to support numeracy-sensitive communication and disease education. More research is needed to elicit additional insights from other patient populations and examine feasibility and efficacy of interventions.

\section{ACKNOWLEDGMENTS}

This research was supported by a grant from the American Kidney Fund Clinical Scientist in Nephrology Program (J. W. N.), and by National Institutes of Health grants to J. W. N. (K23 DK 097183-01A1), T. A. I. (K24 DK62849), C. Y. O. (NIDDK K01 DK087894), and K. L. C. (K23 DK080952-02S1). C. Y. O. is also supported by the Vanderbilt Center for Diabetes Translation Research (P30 DK092986). The authors have no conflicts of interest to report.

Manuscript received August 2014; revised September 2014.

\section{REFERENCES}

1 Institute of Medicine. Health Literacy: A Prescription to End Confusion. Washington (DC), National Academies Press. 2004.

2 Dewalt D, Berkman N, Sheridan S, Lohr K, Pignone M. Literacy and health outcomes: A systematic review of the literature. J Gen Intern Med. 2004; 19:1228-1239.

3 Golbeck AL, Ahlers-Schmidt CR, Paschal AM, Dismuke SE. A definition and operational framework for health numeracy. Am J Prev Med. 2005; 29:375-376.

4 Abdel-Kader K, Dew MA, Bhatnagar M, et al. Numeracy skills in CKD: Correlates and outcomes. Clin J Am Soc Nephrol. 2010; 5:1566-1573.

5 Ando H, Cousins R, Young C. Achieving saturation in thematic analysis: Development and refinement of a codebook 1, 2, 3. Compr Psychol. 2014; 3: p. 7. Article 4.

6 Glaser B, Strauss A. The Discovery of Grounded Theory: Strategies for Qualitative Research. Chicago (IL), Aldine De Gruyther. 1967.

7 Goetz JP, LeCompte MD. Ethnographic research and the problem of data reduction. Anthropol Educ Q. 1981; 12:51-79.

8 Stewart D, Shamdasani P, Rook D. Focus Groups Theory and Practice, Vol. 20, 2nd edn. Thousand Oaks, California, Sage Publications, Incorporated. 2007.

9 Groome PA, Hutchinson TA, Tousignant P. Content of a decision analysis for treatment choice in end-stage renal disease: Who should be consulted? Med Decis Making. 1994; 14:91-97.
10 Zikmund-Fisher BJ, Witteman HO, Dickson M, et al. Blocks, ovals, or people? Icon type affects risk perceptions and recall of pictographs. Med Decis Making. 2014; 34:443-453.

11 Apter AJ, Paasche-Orlow MK, Remillard JT, et al. Numeracy and communication with patients: They are counting on us. J Gen Intern Med. 2008; 23:2117-2124.

12 Schapira MM, Fletcher KE, Gilligan MA, et al. A framework for health numeracy: How patients use quantitative skills in health care. J Health Commun. 2008; 13:501517.

13 Levy H, Ubel PA, Dillard AJ, Weir DR, Fagerlin A. Health numeracy: The importance of domain in assessing numeracy. Med Decis Making. 2014; 34:107-115.

14 Huizinga MM, Carlisle AJ, Cavanaugh KL, et al. Literacy, numeracy, and portion-size estimation skills. Am J Prev Med. 2009; 36:324-328.

15 Norris K, Nissenson AR. Race, gender, and socioeconomic disparities in CKD in the United States. J Am Soc Nephrol. 2008; 19:1261-1270.

16 Mehrotra R, Kermah D, Fried L, Adler S, Norris K. Racial differences in mortality among those with CKD. J Am Soc Nephrol. 2008; 19:1403-1410.

17 Hays J, Hunt JR, Hubbell FA, et al. The Women's Health Initiative recruitment methods and results. Ann Epidemiol. 2003; 13(9 Suppl ):S18-S77.

18 Kennedy TJ, Lingard LA. Making sense of grounded theory in medical education. Med Educ. 2006; 40:101108.

19 Schatell D, Wise M, Klicko K, Becker BN. In-center hemodialysis patients' use of the internet in the United States: A national survey. Am J Kidney Dis. 2006; 48:285291.

20 Cresci MK, Jarosz PA. Bridging the digital divide for urban seniors: Community partnership. Geriatr Nurs. 2010; 31:455-463.

21 Sarkar U, Karter AJ, Liu JY, et al. Social disparities in internet patient portal use in diabetes: Evidence that the digital divide extends beyond access. J Am Med Inform Assoc. 2011; 18:318-321.

22 Chiasson MA, Hirshfield S, Rietmeijer C. HIV prevention and care in the digital age. J Acquir Immune Defic Syndr. 2010; 55(Suppl 2):S94-S97.

23 Spring B, Schneider K, McFadden HG, et al. Multiple behavior changes in diet and activity: A randomized controlled trial using mobile technology. Arch Intern Med. 2012; 172:789-796.

24 Curtin RB, Walters BA, Schatell D, Pennell P, Wise M, Klicko K. Self-efficacy and self-management behaviors in patients with chronic kidney disease. Adv Chronic Kidney Dis. 2008; 15:191-205.

25 Curtin RB, Sitter DC, Schatell D, Chewning BA. Selfmanagement, knowledge, and functioning and wellbeing of patients on hemodialysis. Nephrol Nurs J. 2004; 31:378-386, 396; quiz 387. 
26 Huffman M. Health coaching: A new and exciting technique to enhance patient self-management and improve outcomes. Home Healthc Nurse. 2007; 25:271-274, quiz 275-276.

27 Olsen JM, Nesbitt BJ. Health coaching to improve healthy lifestyle behaviors: An integrative review. Am J Health Promot. 2010; 25:e1-e12.

28 Baker DW. Reading between the lines: Deciphering the connections between literacy and health. J Gen Intern Med. 1999; 14:315-317.

\section{SUPPORTING INFORMATION}

Additional Supporting Information may be found in the online version of this article at the publisher's web-site:

Appendix S1 Structured interview questions-topic areas. 